\title{
Impact of control technology on fight against COVID-19
}

\author{
Zihuan Feng ${ }^{1}$, Xun liang ${ }^{1,}{ }^{*}$, Chengzhen $\mathrm{Zhao}^{2}$, and Jiachen $\mathrm{Wu}^{1}$ \\ ${ }^{1}$ School of information, Renmin University of China, China \\ ${ }^{2}$ School of Economics, Beijing Wuzi University, China
}

\begin{abstract}
Since the outbreak of COVID-19, all countries have taken certain measures to restrain the spread of COVID-19. It is a complex and practical issue to study the measures and effects of the epidemic prevention and control. The spread of the epidemic in different countries has certain characteristics, and the prevention and control models and effects in different countries are also different. In this paper, the key factors affecting the prevention effect of COVID-19 were analyzed by integrating multiple data and using interdisciplinary methods of mathematics, statistics and information management science on the basis of public management, and the system model of influencing factors of COVID-19 was constructed.
\end{abstract}

Keywords: Data mining, Analysis of Internet public opinion, Prevention and control technology.

\section{Introduction}

The outbreak of COVID-19 is a key event affecting the world economy, health and safety. Its spread in various countries has certain characteristics, and the mode and effect of COVID-19 prevention and control in different countries are also different. The development stages of COVID-19 in different countries are different. This is not only related to the level of medical treatment in various countries, but also related to the differences in the corresponding cultural system, climate and environment, COVID-19 information dissemination mode, control policies and guidance modes in relevant departments, and public health emergency response system in various countries[1]. The research on the measures and effects of COVID-19 prevention and control is a complex practical problem. It is not perfect to analyze and deduce only on the theoretical level. It needs to use interdisciplinary research methods to analyze [2-4].

Due to the emergence of modern media tools such as Tiktok and Facebook, the transmission mode, characteristics, speed and prevention and control measures of COVID19 are totally changed. Especially in the context of the current Internet, the rapid development of $5 \mathrm{G}$ technology provides a more convenient channel for information dissemination. Short video as the carrier of information has the characteristics of fast transmission speed, wide audience and wide range. In a short time, it can have tens or even

\footnotetext{
* Corresponding author: xliang@ruc.edu.cn
} 
millions of broadcast volume, and can convey real-time information to citizens of different social occupations and ages. Because the information contains pictures, video and audio, compared with ordinary text information, it is more credible and easier to attract the attention of citizens [5]. COVID-19 information itself has attracted much attention. Once the rapid spread of social media information, it will certainly have a huge impact. Reasonable use of this impact, together with the measures taken by the management and control departments, can play a certain role in guiding citizens through the means of public opinion to help citizens correctly understand the epidemic, strictly implement prevention and control measures, and at the same time, it can also avoid excessive exaggeration of video and other behaviors and citizens' panic. Therefore, in the study of the effect of different institutional models in response to COVID-10, we need to first combine the current form of the Internet, combined with our social media research direction, and take "the role of social media information dissemination in the epidemic" as the "shadow clue", so as to truly analyze the reasons for the difference of COVID-19 effect under different institutional models.

COVID-19 has spread all over the world since its first discovery. COVID-19 is the core of this new multidisciplinary approach. By summarizing and combing COVID-19 patterns, control measures and the final effect in different countries and regions, the relationship between "threshold and epidemic effect" is formed, and the deep mechanism and causes of the difference are further studied. The role of social media information dissemination in the epidemic situation "is the" shadow clue ", and the possible connection and social impact of prevention and control policies and effects under different system modes are analyzed. Taking the global COVID-19 prevention and control as a case, through the interdisciplinary research method, considering a variety of COVID-19 related factors, doing quantitative and qualitative analysis of the critical points of COVID-19 caused by different factors, then the deep-seated connotation is excavated in the summary and classification. At last, from the macro perspective, we summarize the endogenous causes of the prevention and control results. In the current situation that COVID-19 has been basically controlled, we hope to provide practical suggestions for the following COVID-19 prevention and control, urban operation guarantee, resumption of work and production, and post epidemic economic revitalization of the control departments, help the control departments quickly recover and return to the right track after the end of COVID-19, and minimize the adverse impact of COVID-19.

In view of the influence of different institutional cultures, the spreading and diffusion mode of COVID-19 and the handling mode and control effect of COVID-19 are very different, and the reason for the difference is not very clear. Therefore, it is of great theoretical and practical significance to conduct in-depth research on the future policy guidance timely around the "main clue" of COVID-19 prevention mode and effect of different countries in different cultural systems, combined with the background of the current Internet public opinion communication as the "shadow clue", using the interdisciplinary analysis method.

\section{Key influencing factors of COVID-19 prevention effects}

COVID-19 has spread all over the world since its first discovery. On the basis of public management, using the interdisciplinary methods of mathematics, statistics and information management science [6], we carried out data mining and analyze the empirical results of epidemic critical point (threshold). Table 1 shows the epidemic data in a certain area. On February 20, the number of remaining confirmed cases reached the peak, and then the epidemic situation was under control, and the number of remaining confirmed cases began to decline. During the outbreak period of COVID-19, a number of open beds in designated 
hospitals were added every day for patients who needed to be diagnosed. It can be seen from the table that the ratio of retained confirmed patients to the number of open beds was 2.2 at the maximum. Based on this information, the critical point of COVID-19 can be defined as the time when the ratio of confirmed diagnosis to the number of beds available for confirmed patients is equal to 2.2 .

Table 1. Epidemic statistics in a certain area.

\begin{tabular}{cccc}
\hline Date & Retained diagnosis & $\begin{array}{c}\text { Number of open } \\
\text { beds }\end{array}$ & $\begin{array}{c}\text { Retained diagnosis/ } \\
\text { number of open beds }\end{array}$ \\
\hline $2020-02-01$ & 3747 & 6754 & 0.6 \\
$2020-02-02$ & 4686 & 7259 & 0.6 \\
$2020-02-03$ & 5801 & 8199 & 0.7 \\
$2020-02-04$ & 7654 & 8254 & 0.9 \\
$2020-02-05$ & 9305 & 8574 & 1.1 \\
$2020-02-06$ & 10639 & 8895 & 1.2 \\
$2020-02-07$ & 12393 & 9057 & 1.4 \\
$2020-02-08$ & 13496 & 9312 & 1.4 \\
$2020-02-09$ & 15177 & 10300 & 1.5 \\
$2020-02-10$ & 16500 & 12437 & 1.3 \\
$2020-02-11$ & 17361 & 12922 & 1.3 \\
$2020-02-12$ & 29507 & 14269 & 2.1 \\
$2020-02-13$ & 32959 & 15018 & 2.2 \\
$2020-02-14$ & 34289 & 15985 & 2.1 \\
$2020-02-15$ & 35314 & 17262 & 2.0 \\
$2020-02-16$ & 36385 & 18816 & 1.9 \\
$2020-02-17$ & 37152 & 19161 & 1.9 \\
$2020-02-18$ & 38020 & 19927 & 1.9 \\
$2020-02-19$ & 37994 & 19989 & 1.9 \\
$2020-02-20$ & 37448 & 20989 & 1.8 \\
\hline 6010 & 19 & 9.9 \\
\hline
\end{tabular}

We completed the following experiments. The empirical results are based on the following hypotheses: 1) if the ratio is lower than 2.2, it means that the medical resources are sufficient and the diagnosed patients can get the proper treatment. When the ratio is greater than 2.2 , it means that medical resources are in short supply and some confirmed patients can't be treated well; 2) assuming that the ratio is greater than 2.2, the infection rate of confirmed patients will increase correspondingly, the cure rate will decrease correspondingly, and the mortality rate will rise correspondingly, and COVID-19 will be out of control; 3) assuming that the number of beds will increase 750 per day (taking the average value of the actual number of new beds per day from February 1 to February 20). The experimental results are shown in Figure 1. 


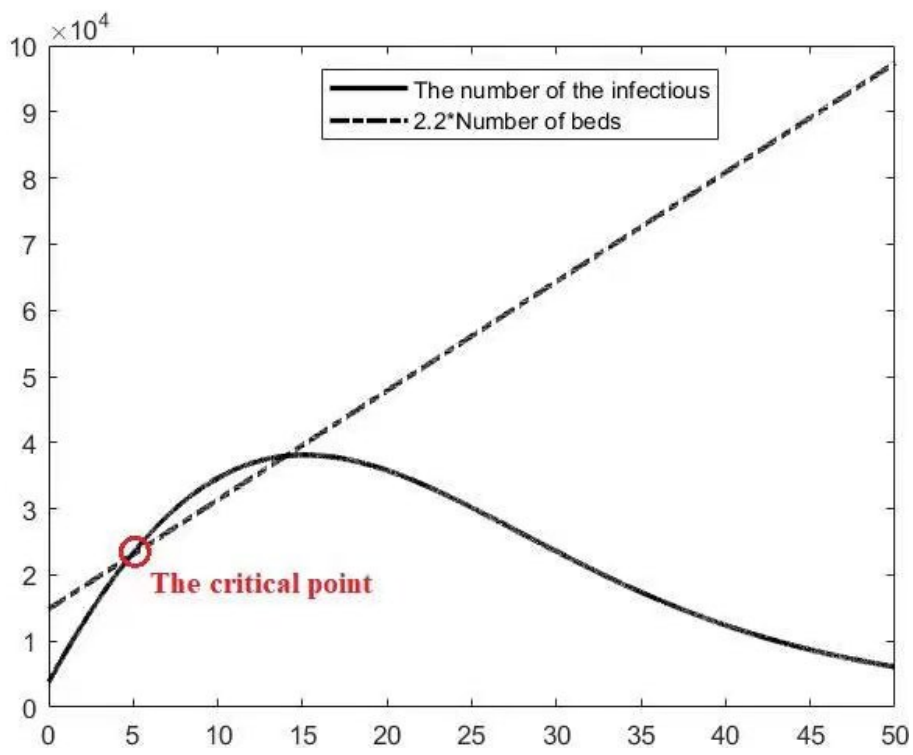

Fig. 1. Trend of the number of confirmed cases when the impact of insufficient beds on the epidemic situation is ignored, and the unit of $\mathrm{x}$-axis is day and $\mathrm{y}$-axis is the number of persons.

Suppose that when medical resources are scarce, the worse case is that the infection coefficient and the isolation degree of the confirmed person is the same as the latent person. In this case, it can be seen from the results in Figure 2 that COVID-19 will be out of control.

From the above analysis, it can be seen that the root cause of the difference in COVID19 prevention effect of different countries is probably the control of the critical point. Beyond this critical point, we will lose control. There are other factors at this critical point, not only the level of medical treatment, but also the factors of culture, population, climate and so on. Among them, the influencing factors can be divided into direct factors and indirect factors. The direct influencing factors include the factors that can be directly observed, such as population density, climate and environment, medical level, intervention level of management and control departments, etc. The indirect factors include the factors that can be transformed from the direct factors through mathematical fitting method, such as the isolation level. The isolation level of citizens is determined by the interaction of several direct factors, such as cultural background, religious belief, control department intervention and so on. In the future, we will analyze the critical point of COVID-19 prevention effect of each country in combination with the epidemic situation and related influencing factors in table 2. 


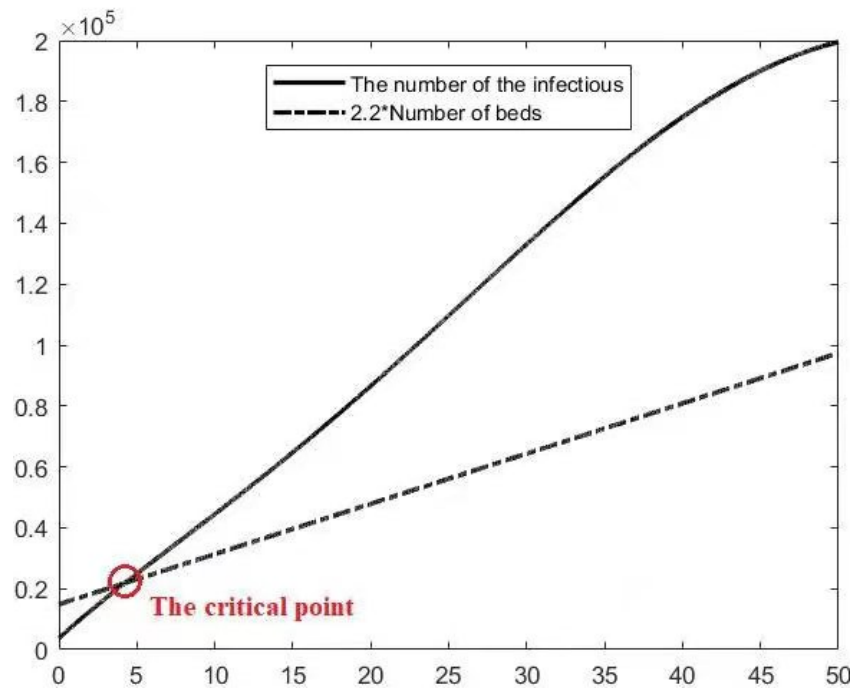

Fig. 2. Trend of the number of confirmed cases retained considering the bad impact of insufficient beds on the epidemic situation, and the unit of $\mathrm{x}$-axis is day and $\mathrm{y}$-axis is the number of persons.

Table 2. Epidemic situation and related influencing factors in different countries

\begin{tabular}{|c|c|c|c|c|c|c|c|}
\hline County & $\begin{array}{l}\text { Cumulative } \\
\text { number of } \\
\text { confirmed } \\
\text { cases } \\
(10000)\end{array}$ & $\begin{array}{c}\text { Number of } \\
\text { cured } \\
(10000)\end{array}$ & $\begin{array}{c}\text { Cure rate } \\
(\%)\end{array}$ & $\begin{array}{c}\text { Population } \\
\text { density } \\
\text { (population / } \\
\mathrm{km} 2 \text { ) }\end{array}$ & $\begin{array}{c}\text { Medical } \\
\text { level } \\
\text { (HAQ index) }\end{array}$ & $\begin{array}{c}\text { Epidemic } \\
\text { intervention } \\
\text { level of control } \\
\text { departments (1- } \\
3 \text { ) }\end{array}$ & Climate type \\
\hline America & 172 & 47 & 27.33 & 33 & 89 & 1 & varied \\
\hline Brazil & 39 & 15 & 38.46 & 24 & 64 & 2 & tropic \\
\hline Russia & 37 & 14 & 37.84 & 9 & 75 & 2 & $\begin{array}{c}\text { the temperate } \\
\text { zone }\end{array}$ \\
\hline Spain & 28 & 19 & 67.86 & 92 & 92 & 2 & varied \\
\hline Britain & 26 & 0.11 & 0.42 & 271 & 90 & 1 & $\begin{array}{c}\text { Temperate } \\
\text { Oceanography }\end{array}$ \\
\hline Italy & 23 & 14 & 60.87 & 201 & 95 & 2 & varied \\
\hline France & 18 & 6 & 33.33 & 123 & 92 & 1 & $\begin{array}{c}\text { Temperate } \\
\text { Oceanography }\end{array}$ \\
\hline Germany & 18 & 16 & 88.89 & 232 & 92 & 3 & $\begin{array}{l}\text { Transitional } \\
\text { climate } \\
\text { between } \\
\text { oceanic and } \\
\text { continental } \\
\text { climate }\end{array}$ \\
\hline Turkey & 15 & 12 & 80.00 & 105 & 74 & 3 & varied \\
\hline India & 15 & 6 & 40.00 & 404 & 41 & 2 & $\begin{array}{l}\text { Tropical } \\
\text { monsoon }\end{array}$ \\
\hline
\end{tabular}

Under the active control of national control departments, combined with the local medical level, COVID-19 has been basically controlled, but the potential risks are relatively large. Any country out of control is likely to lead to a global pandemic. The number and distribution of population are obviously highly correlated with COVID-19.

\section{Analysis on differences of COVID-19 prevention effect caused by control mode}

Today, any metropolis in the world sends hundreds of thousands of tourists to all parts of the world every day. From horses to sailboats, to railways, to cars, and then to airplanes, 
human beings are speeding up their travel, but at the same time, the spread of the virus is also speeding up. Nowadays, the trend of trade globalization is becoming more and more intense, and frequent travel has followed. As many people have said, the emergence of such events like COVID-19 is only a matter of time. From the perspective of the transmission mode and path of each country, although each country has its own characteristics and rules, generally speaking, they are basically " The first case is input type and the diffusion is because of accumulation ", that is, the first confirmed cases are all returned from other countries, but it has not attracted the attention of the country and the aggregation activities are not reduced, then leading to large-scale diffusion [7].

This section will deeply analyze the control mechanism of the responsibility faced with COVID-19, the medical management system and the relationship between the two, based on the research of COVID-19 prevention effect in the world, using the method of induction and summary, combined with the background and culture of each control area. Combined with the transmission mode of COVID-19 information under the condition of Internet, we will also analyze the influence of public opinion on COVID-19 control. The specific contents include:

(1) We analyzed the performance of the responsibility mechanism of management and control departments under different cultural backgrounds in the face of such major public health emergencies, and analyzed the harm and impact path of the lack of social responsibility.

(2) Taking the relationship of "factor threshold - COVID-19 prevention effect" as the "main clue", we combine the COVID-19 prevention measures and the COVID-19 prevention effect of various regions with the responsibility mechanism of control departments to find out the interests.

(3) Combined with the cultural and institutional background of domestic and foreign countries, taking "the role of social media information dissemination in the epidemic" as the "shadow clue", we analyzed the regulatory role that the management and control departments can play in the guidance of public opinion. Taking public opinion as the COVID-19 prevention weapon, we analyze the changes of citizens' psychological state and coping behavior caused by public opinion.

(4) We construct the effect evaluation system and model of responsibility mechanism of management and control departments in response to major public health emergencies. Based on novel corona virus pneumonia theory, from the perspective of stakeholders, we study the response and control responsibility index system of major public health emergencies taking COVID-19 as an example. It sets up the index system from several dimensions, selects representative indicators in each dimension, and identifies two levels of indicators under several dimensions, finally constructs a complete index system and evaluation contents.

The severity and speed of COVID-19 in each country are closely related to its cultural characteristics and political system.

From the above summary, the control departments' inaction and the public's noncooperation are the difficulties of COVID-19 prevention and control. However, from the perspective of the current Internet dissemination of social we media information, it may provide a solution to this pain point. From the perspective of public opinion, we should correctly guide people's psychology and behavior, because public opinion sometimes has more persuasive and practical effect on people than the policy control. Especially in the current situation that most people spend a lot of time on mobile phones and computers, and small video applications are very popular, the information spread on the Internet can affect more people faster. 


\section{Conclusion}

Under the shadow of COVID-19, the countries are almost "economically" "stopped" to deal with catastrophe. Judging from the current situation, globally, it still needs to wait when the economy will end its "pause" to restore normal production and living order. Various national control departments have also introduced their own control measures, but the effects are different. We can see that although developed countries have advanced medical equipment and sufficient economic strength, the degree of epidemic control may not be related to the degree of development of the country. Although they have advanced medical level and rich medical resources, if they can't make effective use of their own advantages, they will also provide opportunities for the spread of the virus in a large area. The process of epidemic prevention and control must be time-consuming, laborious and costly, which will have a certain negative impact on the country's economic development. However, if it can't be effectively controlled in the early stage, the economic and trade losses caused to a country will be long-term: once the inflation caused by the failure of factories and enterprises to return to work and hoarding of goods by the whole people occurs, it will cause huge pressure to the country.

In this kind of emergency, the degree of response and response measures are the key to effectively control its development. Especially in the event that may endanger the vital interests of the people, the management and control departments of all countries should first put the national interests first, appease the people, and minimize the possible losses as far as possible under the situation of unavoidable impact on national development. The next step of research should closely follow the development of COVID-19, after the global COVID-19 is basically stable and reaches the inflection point, we should further analyze the global COVID-19 transmission mode and give the analysis combined with data and theory as the basis and reference for future prevention and response to such emergencies.

This work was supported by the National Social Science Foundation of China (18ZDA309), the National Natural Science Foundation of China (62072463, 71531012), the Opening Project of State Key Laboratory of Digital Publishing Technology of Founder Group (413217003).

\section{References}

1. Novel coronavirus pneumonia epidemic situation and coping strategies. Retrieved April 10, 2020 from http://www.chinado.cn/?p=9200

2. Liang Ma, Mei Yang, Chuan Ai, et al. Evaluation of the effectiveness of COVID-19 epidemic control measures based on ACP method [J]. Journal of Intelligent Science and Technology,2020,2(01):88-98.

3. Chengzhuo Li, Wentao Wu, Zhenyu Pan, et al. Control effect analysis of COVID-19 in Zhejiang Province based on SIR model and basic regenerative number [J]. Zhejiang medical,2020,42(04):311-314.

4. Baosen Wang, Qiming Fan. Influences of human rights and institutions on prevention and control efficiency under major outbreak [J]. Economic Research Guide,2020(23):144-146.

5. Bo Cheng. Public opinion communication characteristics of public health emergencies in the era of omnimedia [J]. Audio-visual,2020(04):114-115.

6. Tianmu Chen, Zeyu Zhao, Jia Rui, et al. Calculation of COVID-19 population transmission capacity and simulation evaluation of prevention and control measures in Xiamen City $[\mathrm{J}]$. Journal of Xiamen University (Natural Science Edition),2020,59(03):298-303. 
7. Jingyu Chen, Yetao Luo, Xiaohua Liang, et al. The influence of policy on control effect in major public health emergencies: A case study of COVID-19 epidemic prevention and control in Chongqing $[\mathrm{J}]$. Journal of Pediatric Pharmacy,2020,26(04):28-31. 